\title{
物联网服务建模: 一种基于环境建模的方法
}

\author{
(1) 北京大学信息科学技术学院软件研究所, 北京 100871 \\ (2) 北京大学高可信软件技术教育部重点实验室, 北京 100871 \\ (3) 中国科学院数学与系统科学研究院, 北京 100190 \\ (4) 中国科学院大学, 北京 100049 \\ (5) 北京信息科技大学计算机学院, 北京 100085 \\ * 通信作者. E-mail: zhijin@sei.pku.edu.cn
}

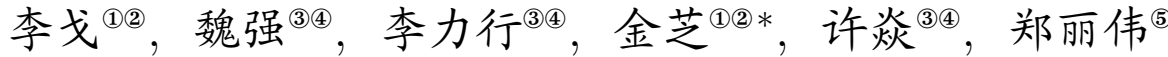

收稿日期: 2013-05-08; 接受日期: 2013-08-19

国家重点基础研究发展计划 (批准号: 2011CB302704) 和国家自然科学基金 (批准号: 61232015) 资助项目

\begin{abstract}
摘要 物联网服务是传统 Web 服务通过传感器网络向物理环境的延伸, 它通过传感器网络感知物 理环境中的实体, 也向物理环境实体施加作用。与传统 Web 服务相比，由于物联网服务受到所依赖 的物理环境的时间受限性、资源受限性和设备潜在故障概率的影响, 物联网服务的响应速度、服务 能耗和容错能力等特性成为影响物联网系统整体特性的重要因素. 因此, 对物联网服务进行全面建 模, 对物联网服务所处的外部环境进行形式化描述, 并结合物理环境模型对物联网服务的性质进行 分析, 对于确保物联网系统的正确性、稳定性非常必要. 本文针对物联网服务的特点, 结合基于环境 建模的需求工程思想, 提出一种基于环境的物联网服务三元问题域建模框架, 给出了物联网服务建 模本体以及相应的建模原则. 在此基础上, 提出了一种物联网服务行为建模方法, 该方法将物联网服 务和物理环境建模为概率时间自动机，将用户期望的服务特性描述为时序逻辑公式，为物联网服务 功能行为正确性验证和非功能性约束可满足性验证奠定了基础.
\end{abstract}

关键词 物联网 物联网服务 环境建模 服务建模本体 服务建模过程

\section{1 引言}

物联网 (IoT, Internet of Things) 是新一代信息技术的重要组成部分, 是物物相连的互联网. 它将 互联网扩展到物理世界中去, 通过 RFID(radio-frequency IDentification), 传感器, 全球定位等信息传感 技术, 按约定的协议, 把物理世界中的物体与互联网相连接, 进行信息交换和通信, 从而实现对物体的 智能化识别、定位、跟踪、监控和管理 ${ }^{[1]}$.

面向服务架构 (SOA, service-oriented architecture) 将异构平台上的应用程序的不同功能单元封 装为具有良定义接口和规范的服务, 并以松耦合的方式整合在一起, 能有效的解决资源重用和异构组 件之间的互操作性问题. 近年来, 许多研究者开始关注于将面向服务的思想应用于物联网中, 引出了 对物联网服务的研究 ${ }^{[2 ~ 4]}$. 其基本思想是: 把物理世界中各种设备的功能服务化, 称为现实世界服务 (real-world services) 或设备服务 (device services), 这些设备服务以一种统一的接口向外界提供设备的 
功能, 使各种异构的设备之间, 设备与传统 Web 服务之间可以互相调用, 进行信息传递, 同时设备服 务还可以被动态发现与组合, 从而实现物理世界与虚拟世界的无缝整合. 本文将通过传感器网络和互 联网连接在一起并用于实现特定业务能力的设备服务与传统 Web 服务一起统称为物联网服务 (IoT services, services in the Internet of Things).

从服务提供的角度看, 物联网服务具有不同于传统 Web 服务的特点. 首先, 物联网服务大都直接 通过物理设备与现实世界发生交互, 其操作环境具有自主变化的特点. 其次, 与物联网服务交互的物 理环境的时间性、资源受限性和可能的设备故障, 对物联网服务提出了响应速度要求, 以及服务部署 能耗分析、和容错等能力要求. 传统的 Web 服务的建模和分析方法, 一般忽略对其操作环境的建模, 不能直接将它们用于物联网服务的建模和分析.

本文在分析和总结物联网服务特点的基础上, 认识到与物联网服务进行交互的物理实体模型对进 行物联网服务的建模及其功能和非功能性质的分析具有至关重要的作用, 结合基于环境建模的需求工 程思想, 提出基于环境模型的物联网服务建模框架. 然后本文设计了一个物联网服务本体, 以规范建模 框架中的概念和关联关系, 并总结出一组建模原则. 在此基础上, 本文提出一个物联网服务建模过程, 该建模过程强调对物理环境的刻画, 以及服务与环境的交互以体现物联网服务的功能行为描述. 它在 关注服务功能行为的同时，建模多种服务质量属性 (时间属性、可靠性、资源消耗), 并基于概率时间 自动机 (probabilistic timed automata) 和时序逻辑 (temporal logic) 进行形式化的模型解释, 为进行物 联网功能行为正确性验证和非功能性约束可满足性验证奠定基础.

本文其余部分内容组织如下. 第 2 节从物联网服务描述, 体系架构和建模等几个方面介绍相关工 作; 第 3 节针对物联网服务的特点, 提出物联网服务建模和分析框架, 并给出物联网服务建模的本体 及建模原则; 第 4 节给出基于环境的物联网服务建模过程; 第 5 节利用具体案例阐述物联网服务建模 过程; 第 6 节总结全文, 讨论进一步的研究方向.

\section{2 相关工作}

与本文研究内容相关的研究工作主要包含物联网服务描述、体系结构和服务建模三个方面. 在物 联网服务描述方面, Guinard 等人 [4] 提出可采用轻量级的面向服务范型来服务化物联网中资源受限 设备的功能, 并给出了两种轻量级服务描述方法, 即基于 DPWS (devices profile for web services) 的服 务描述方法, 和将资源受限设备的功能封装为 RESTful Web 服务. Pautasso 等人 [5] 总结了 DPWS 和 RESTful Web 服务各自的优劣, 认为 RESTful Web 服务更加轻量、可扩展性强, 对于开发者而言容易 理解、学习和实现, 非常适合于物联网系统中的程序设计, 但安全性较低. 而 DPWS 理解较难, 但其 继承自 WSDL(web service description language), 能满足更高的安全性需求, 具备更好的抽象层次.

在面向服务的物联网体系架构方面, 越来越多的研究关注于如何将面向服务的方法应用于物联网 系统体系架构的构建. SODA (servic-oriented device architecture) ${ }^{[2]}$ 是 IBM 的 Deugd 等人提出的一个 物联网服务体系架构, 其目标在于将各种物理设备提供的服务连接到企业服务总线 (enterprise service bus）中, 使得用户能够象访问 Web 服务一样访问设备服务. 其不足之处在于未提供对设备的状态监 视和维护, 导致服务提供的可靠性得不到保障. SOCRADES(service-oriented cross-layer infrastructure for distributed smart embedded devices $)^{[4]}$ 是由 SAP 研究院提出的一种基于 Web 服务的制造车间集 成架构, 其目标是借助 Web 服务技术将制造车间中的智能化设备同高层后台系统紧密联系起来, 实现 对设备的实时监测和控制, 提高生产效率和安全保障. 其不足之处在于所考虑的上下文信息类型单一, 
比如未考虑时间、能耗等相关上下文信息, 也未提供有效的物联网服务性能分析方法. Buckl 等人 ${ }^{[3]}$ 提出了服务网关 (service gateway) 的概念, 服务网关将信息处理的代价从资源受限的物理设备转移到 资源丰富的网关上，更适合于那些资源匮乏的设备. 但是因为服务网关中存储的信息相对静止，所以 并不适合于设备连接关系频繁动态变化的应用场景.

在物联网服务建模方面也已经有了一些初步工作. 英国 Surrey 大学的 Barnaghi 等人 ${ }^{[6]}$ 提出一种 关于物联网服务的语义建模方法. 他们提出了一组物联网语义模型中的核心概念 (设备, 实体, 资源, 服务) 及其相互关系, 并采用 OWL-DL 描述实体和资源, 采用 OWL-S 来表示物联网服务, 使服务与 实体、资源紧密关联在一起. 该建模方法不仅为物联网中各类对象提供了一个统一的接口描述, 而且 通过语义信息标注, 使得服务描述变得机器可理解, 从而提高服务自动发现的正确性. 然而目前这方 面的工作还比较初步, 存在一些不足, 比如, 对物联网服务功能的描述采用经典的 IOPE, 并未考虑服 务的行为或业务流程, 导致无法分析复杂的服务组合匹配; 没有提供服务质量的建模和分析方法, 从 而无法刻画物联网服务非功能性约束的可满足性.

我们之前的工作提出以时间自动机和时序逻辑 TCTL(time computation tree logic) 分别表达物联 网服务与时间相关的行为和性质, 用模型检测工具 UPPAAL 对物联网服务的时效正确性进行验证 ${ }^{[7]}$. 我们还提出以马尔可夫决策过程和概率时序逻辑 PCTL(probabilistic computation tree logic) 分别描 述物联网服务与可靠性和资源消耗相关的行为和性质 ${ }^{[8]}$, 用概率模型检测工具 PRISM 进行验证和分 析, 以选择出符合特定可靠性和资源消耗需求的物联网服务组合策略. 但未涉及物联网服务和设备服 务的统一描述和分析方法.

\section{3 基于环境的物联网服务建模}

本课题组之前的工作曾提出基于环境的 Web 服务建模和分析方法 ${ }^{[9,10]}$. 其主要思想是通过环境 模型定义 Web 服务的行为描述, 赋予 Web 服务在现实世界中的语义信息, 实现了对服务提供和服务 请求的统一表示, 为进一步的 Web 服务发现和自动组合提供了良好的语义基础. 本文工作在此基础 上, 通过分析物联网服务的特征, 提出将设备服务集成到基于环境实体建模的方法中, 为 Web 服务和 设备服务提供统一的描述和分析手段, 同时分析出物联网服务的典型非功能性约束, 以便能在服务功 能描述和分析的同时支持服务质量约束的描述和分析.

\section{1 物联网服务建模的三维视角}

目前已经存在一些基础的物联网系统, 比如用于森林生态监测的 “绿野千传” 物联网系统 ${ }^{[11]}$, 由 美国弗罗里达大学开发的智能家居系统 Gator Tech Smart House ${ }^{[12]}$, 由德国人工智能研究中心主导的 物联网智能工厂项目 SmartFactory ${ }^{\mathrm{KL}[13]}$ 等.

物联网服务系统是人造系统, 人造系统均是人们为了达到某种目的而构建的系统, 需要满足用户 的期望, 实现用户期望它实现的业务逻辑. 物联网服务是这个人造系统中的一种软件服务, 它实现特 定的业务逻辑, 并通过物联网系统将业务逻辑实施到可感知和可控制的物理环境上. 相比于 Web 服 务, 物联网服务有其如下特有的性质:

- 对物理环境的依赖性: 物联网服务的交互对象为物理世界中自主变化的实体. 即直接部署于物 理环境中的物联网服务, 同自主变化的环境实体进行直接交互, 实时采集环境中的数据, 并根据数据 分析的结果, 对环境实体实施适当的作用. 由于环境实体的自主性和动态可变性, 物联网服务的行为 
需要随着环境实体的变化而及时调整.

- 与物理环境的强交互性: 物联网服务的另一个特点体现在它和环境实体的直接交互上. 物联网 服务和环境实体之间的交互是双向的, 即对环境的感知和对环境的作用. 对环境的感知表示物联网服 务监测环境的能力, 对环境的作用体现在物联网系统在分析环境状态的基础上, 控制环境实体去改变 环境, 以满足用户期望的能力. 其中, 对环境的感知表现为由各类感知设备所提供的物联网服务实时 获取物理环境的各项参数 (如室内温度、湿度, 空调的运行状态等) 的能力. 对环境的作用表现为物联 网服务感知到物理环境的状态变化后, 经过数据分析, 根据一定的业务流程通过执行器控制物理设备 施加作用于环境, 达到调节物理环境的状态, 满足用户期望的能力.

- 行为特性受物理实体性质的约束: 由于大部分物联网服务是实时的、设备相关的、和资源受限 的, 物联网服务和环境的交互需要满足相应的约束:

一 实时性: 物联网服务需要实时关注物理世界的状态, 提供实时服务. 它利用分布广泛的传感网 网络和各种不同类型的传感器, 实现对物理实体的实时状态获取和特定行为的监控, 如通过二氧化碳 传感器监测某一区域的二氧化碳浓度, 利用 GPS 实时跟踪车辆的位置等. 传感器往往按一定的频率 周期性地采集环境实时信息, 不断及时更新数据. 因此, 物联网服务的行为是时间相关的. 此外, 物联 网服务的很多应用场景有非常高的安全性要求, 而这些安全性需求大都与时间紧密相关, 在时间上较 小的偏差, 都可能产生极为严重的后果. 如火灾发生后, 警报的及时发送和相关应急设备的及时响应.

一 资源约束: 与传统的 Web 服务提供设备 (主要为计算机) 不同, 在物联网服务系统中, 由于部 署环境和成本的原因, 服务提供设备无法连接上稳定的电源, 而需要依靠电池供电, 从而导致设备资 源受限 (resource-constrained), 即通信、计算、存储等能力不足. 在这种资源有限的情况下, 为了保证 服务的稳定提供和服务质量, 能耗分析和服务合理部署成为重要关注点.

一 可靠性: 提供物联网服务的设备往往部署于物理环境中, 多采用无线自组织的方式互连, 存在 多种原因使得物联网服务提供具有动态性和不稳定性：(1) 服务提供设备资源受限, 在能量不足的情 况下, 将使得服务提供质量下降甚至失效; (2) 各种移动终端, 如手机、PDA、平板电脑等通过无线方 式互连, 各种服务运行于这些移动设备上, 同时也可以被其他设备所调用, 导致服务的提供者和服务 的使用者都可能动态变化; (3) 部署野外自然环境中的物联网设备, 受自然环境的影响 (如降雨、野生 动物破环等), 可能无法正常提供服务. 如何在这样动态变化的环境中, 发现合适的服务并获取稳定的 服务提供成为重要关注点.

可以看出, 物联网服务系统包含感知流、控制流和业务流三类信息流. 其中,

- 感知流: 由各类感知设备所提供的物联网服务实时获取的物理环境数据;

- 控制流: 由各类物联网控制设备提供的服务向物理环境实体发送的控制指令;

- 业务流: 物联网服务之间的信息交互, 如相互调用和组合, 用于实现物联网服务系统要实现的业 务逻辑.

这三类信息流相互独立, 但同时又构成了物联网服务和物理环境之间的信息流环路, 三者有机地 协调, 共同完成整个物联网服务系统对物理环境的感知和作用. 因此, 本文总结出物联网服务系统建 模的三维视角, 如图 1 所示.

其中, 物理环境为需要物联网服务感知和控制的现实世界部分. 用户需求刻画用户期望的由物联 网服务带来的在现实世界部分中产生的作用和效果. 物联网服务系统包含三类服务: (1) 感知型服务, 用于实时采集物理环境的状态信息; (2) 控制型服务, 通过向物理环境中的可控实体发出指令, 使物理 环境发生期望的变化; (3) 业务逻辑型服务, 根据一定的业务流程协调其它服务的执行, 实现用户的期 


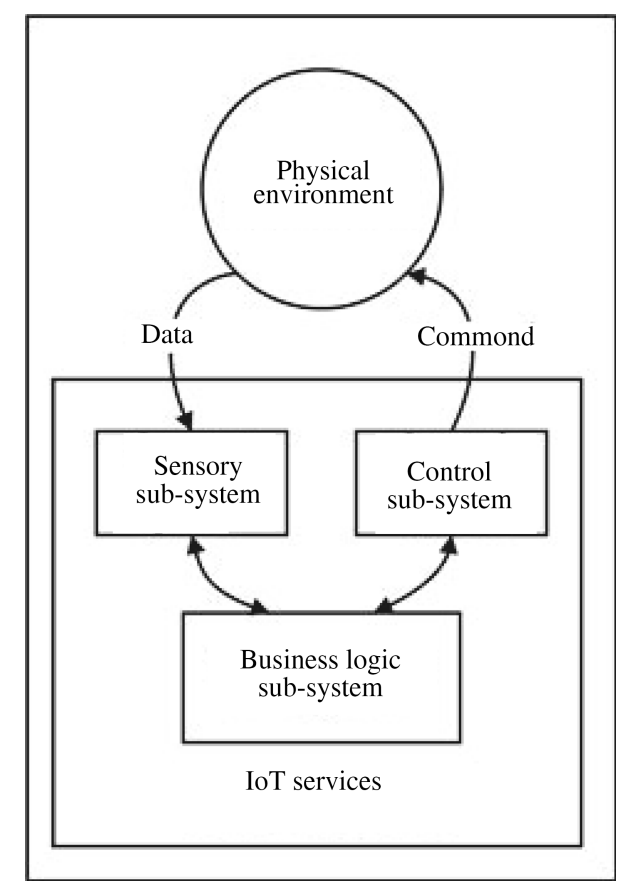

图 1 物联网服务、物理环境、用户需求的关系

Figure 1 Relations among IoT services, environment and user requirements

望. 这种划分实现了物联网服务的感知流、控制流和业务流的分离和协调.

这个建模视角将传统软件建模方法中由软件系统、用户需求组成的二元问题域, 扩展为由物联网 服务、物理环境、期望的性质组成的三元问题域. 物联网服务、物理环境、期望的性质这三者之间的关 联关系可用如下公式表达:

$$
\text { 物联网服务, 物理环境 } \rightarrow \text { 期望的性质, }
$$

该公式所表示的含义是: 若将物联网服务运行于特定物理环境中, 并与之发生交互作用, 则环境发生 的变化将满足期望的性质. 其中, 物联网服务和物理环境的交互体现出物联网系统的目标 - 信息世界 与物理世界的无缝融合. 一方面, 物联网服务的行为可以在它的运行环境中被观察和评估, 服务行为 的作用结果将映射到其所处的环境之上, 另一方面, 物理环境的变化是由环境实体的自主行为以及物 联网服务对环境实体的控制所共同引起的.

\section{2 物联网服务本体}

本体 (Ontology) 是共享概念模型的显式的形式化规格说明 [14]. 本体通过对概念及其相互关系进 行规范化描述, 构建出某一领域的基本知识体系, 提供对该领域知识的共同理解, 确定该领域内共同 认可的词汇, 并从不同层次的形式化模型上给出这些词汇和词汇之间相互关系的明确定义.

从上述物联网服务建模的三维视角出发, 并以通用本体规范为依据, 我们定义了一个物联网服务 系统的本体, 为后续的物联网服务建模提供依据. 下面将详细介绍该本体各组成部分的具体内容.

\subsection{1 概念及其层次结构}

物联网服务本体中的主要概念及其层次结构如图 2 所示, 其中, $\mathrm{T}$ 表示全域概念. 


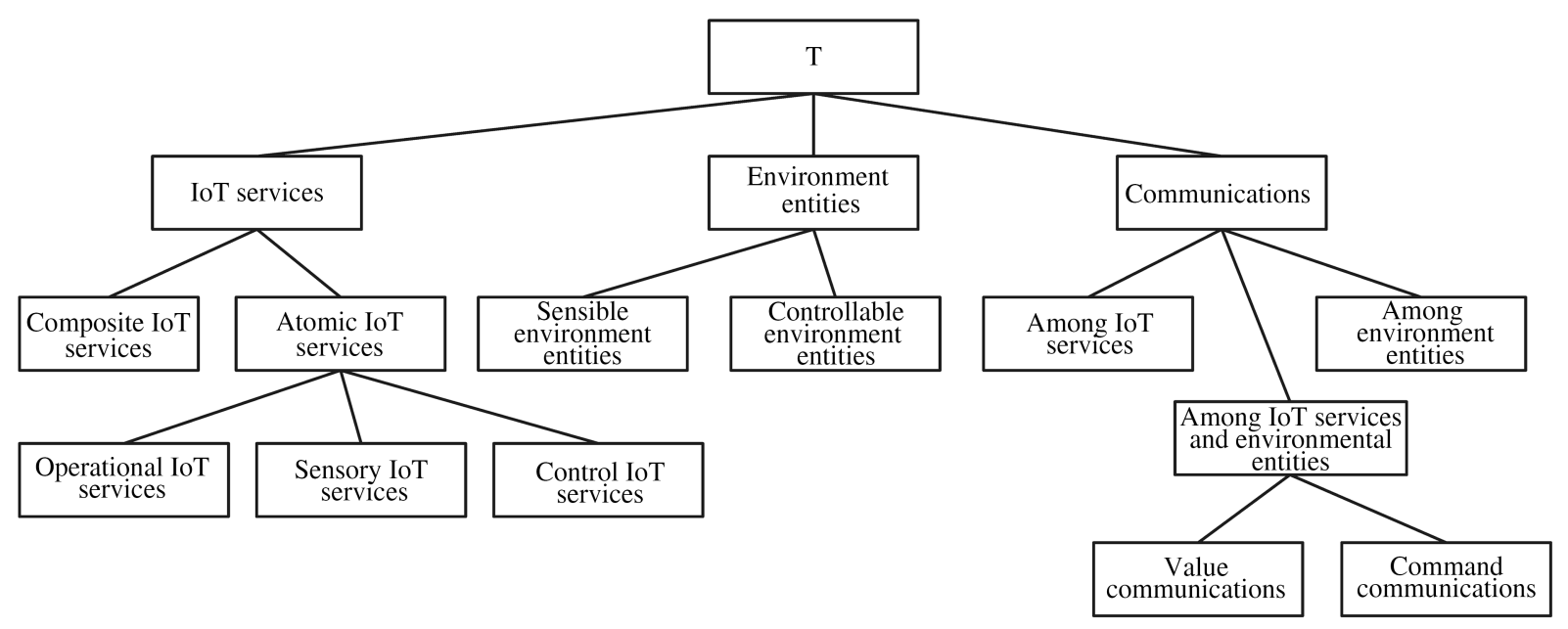

图 2 物联网服务本体概念层次结构

Figure 2 Concept hierarchy of the ontology for IoT Services

主要概念的含义解释如下:

- 物联网服务是指由各类物联网设备提供的、展现设备功能的、可被相互调用的软件模块. 物联 网服务包括原子服务和组合服务:

一物联网原子服务是指服务的实现自包含, 在执行时不可分割, 不可分解为更细粒度的服务. 原 子服务包括以下三类:

1) 感知型服务通过与环境实体的交互, 采集信息, 感知外部环境实体的状态变化, 并能将获取的 信息传输给其它服务. 通常情况下, 感知型服务周期性地采集被感知环境实体的信息, 并且单个感知型 服务只提供对一类环境实体的某项属性的感知能力;

2) 控制型服务首先从其它服务接收到对某个环境实体的控制信息, 然后将这些信息转换为环境实 体可以接受的控制命令, 并将其发送给相应的实体;

3) 处理型服务把从其它服务中获取的信息进行综合处理, 也可将信息传输到其它服务. 注意处理 型服务并不与环境实体直接交互, 相比于设备服务, 它们具有更强的计算能力和存储能力. 处理型服 务也可被看作是轻量级的 Web 服务, 它与 Web 服务的不同之处在于服务提供者是本地设备, 而不是 互联网上的某台计算机.

一 物联网组合服务是指根据特定的业务流程, 由多个原子服务聚合而成的满足用户不同应用需 求的、新的、更复杂的物联网服务.

- 环境实体是组成物理环境的基本元素, 每个环境实体都具有唯一标识, 拥有一定的物理或虚拟 属性, 具有通信能力或允许属性值被感知. 从与物联网服务交互的角度出发, 环境实体可分为以下两 大类:

一 被感知的环境实体可被物联网服务以感知的方式获取其状态值, 但服务无法直接改变这些实 体的状态, 实体的状态以一种自主的、无法被外界准确预知的方式变化着;

一 被控制的环境实体不仅其状态值可以被服务获取, 并且服务可以向这些环境实体发送事先定 义好的指令, 改变实体的状态, 从而控制实体的状态变化.

- 交互刻画出物联网服务与环境实体的动态行为的关联关系, 其可分为以下三类: 
一物联网服务的交互是指物联网服务之间的消息传递、互相调用, 多个服务通过这种交互来实现 协作, 从而完成复杂的任务.

一环境实体的交互是指环境实体之间的相互作用和影响. 这种交互是环境实体的自主行为, 无法 被物联网服务所控制.

一 物联网服务与环境实体的交互是指物联网服务能够感知和作用于环境实体, 并可能导致环境 实体发生期望的变化. 这种交互由物联网服务所主导, 其可进一步分为值交互和指令交互. 其中, 值交 互是指物联网服务获取到被感知的环境实体各项属性的取值; 指令交互是指物联网服务向被控制的环 境实体发出指令, 改变其状态.

\section{2 .2 概念关联和概念属性}

概念不是孤立存在的, 它们之间存在关联关系, 其中每个关联都是一个二元关系, 包括定义域和值 域. 关联中的定义域和值域分别对应本体中的一个概念. 定义关联使得定义域和值域中的元素建立起 了特定联系.

为进一步刻画物联网服务的行为和服务质量属性, 本文引入状态机本体. 该本体中最核心的概念 是有限状态机. 有限状态机的组成部分包括状态、动作、状态迁移. 有限状态机之间可以进行通信 (对 应关联 communicate). 状态迁移表示从一个源状态 (对应关联 from) 到目的状态 (对应关联 to) 的 迁移. 状态迁移由特定事件所触发 (对应关联 trigger), 而且往往伴随着一个动作的执行 (对应关联 has_Action), 同时在迁移中也可引起其它事件 (对应关联 cause). 状态迁移约束用于从非功能的角度 约束状态迁移, 其子概念包括时间约束、可靠性约束、代价约束.

物联网服务系统本体中的重要关联及其含义如表 1 所示.

物联网服务系统本体的概念和关联共同构成了关于物联网服务系统的概念图, 如图 3 所示.

\section{3 建模原则}

根据上述分析, 本文归纳出物联网服务的建模原则:

1) 基于行为规约的统一服务建模原则. 物联网服务的范畴很广, 既含有以 DPWS 或者 RESTful API 描述的设备服务, 又涵盖传统的 Web 服务以及使用各种形式封装的软件功能模块. 尽管各种服 务具有不同的描述语言、通信协议、调用方式等实现细节, 但在系统设计和分析阶段, 需要屏蔽掉这些 差异, 对所有的物联网服务以一种统一、简洁和抽象的方式进行建模. 本文从服务的行为和服务质量 两方面表示物联网服务. 服务行为关注服务对物理环境的感知和作用, 以及服务之间的消息传递、并 发和协作行为的描述; 服务质量属性依附于服务行为之上, 用于描述对服务行为执行的各种约束, 本 文重点建模的服务质量属性包括时间属性、可靠性和资源消耗.

2) 物理环境实体的显式表示原则. 由于物理环境对于物联网服务描述的重要性,需要在建模过程 中将环境以显示的方式表示出来. 环境由各种环境实体构成, 环境实体包括自然物质、物理设备和人. 自然物质的状态可被物联网服务感知, 但一般具有自主的行为, 比如空气、流水、植物、动物等; 物理 设备的状态和行为完全可以被物联网服务所控制, 比如空调、电视机、报警器等; 人, 一般具有自主意 识和目的, 行为难以被预测, 但部分生理状况可被感知.

与物联网服务一样, 在建模中, 环境实体同样也要求以一种统一的形式表达. 每个环境实体既含有 用于强调实体自身属性和实体间的组织结构的数据部分, 又具有动态行为. 环境实体可以通过有限自 动机进行建模，其中，自动机的状态记录环境实体的各项属性值，状态间的变迁则描述环境实体的 


\section{表 1 物联网服务系统本体的关联及其含义}

Table 1 Relationships in the ontology for the system of IoT services

\begin{tabular}{|c|c|c|c|}
\hline Relationship & Domain & Range & Relationship explaination \\
\hline sense & IoT services & Environment entities & $\begin{array}{l}\text { IoT Services perceive the status of } \\
\text { environment entities. }\end{array}$ \\
\hline affect & IoT services & Environment entities & $\begin{array}{l}\text { IoT Services impact the status of } \\
\text { environment entities. }\end{array}$ \\
\hline compose & $\begin{array}{l}\text { Atomic IoT } \\
\text { services }\end{array}$ & Composite IoT services & $\begin{array}{l}\text { Composite IoT services are composed } \\
\text { of multiple atomic services. }\end{array}$ \\
\hline participate & IoT services & $\begin{array}{l}\text { Communications among IoT } \\
\text { services }\end{array}$ & $\begin{array}{l}\text { IoT services communicate with } \\
\text { other services. }\end{array}$ \\
\hline participate & $\begin{array}{l}\text { Environment } \\
\text { entities }\end{array}$ & $\begin{array}{l}\text { Communications among } \\
\text { environment entities }\end{array}$ & $\begin{array}{l}\text { Environment entities communicate } \\
\text { with other entities. }\end{array}$ \\
\hline participate & IoT services & $\begin{array}{l}\text { Communications among IoT } \\
\text { services and environment entities }\end{array}$ & $\begin{array}{l}\text { IoT services communicates with } \\
\text { environment entities. }\end{array}$ \\
\hline participate & $\begin{array}{l}\text { Environment } \\
\text { entities }\end{array}$ & $\begin{array}{l}\text { Communications among environment } \\
\text { entities and IoT services }\end{array}$ & $\begin{array}{l}\text { Environment entities communicates } \\
\text { with IoT services. }\end{array}$ \\
\hline has_Beh & IoT services & Finite state machine & $\begin{array}{l}\text { The behavior of IoT services are } \\
\text { represented using FSM. }\end{array}$ \\
\hline has_QoS & IoT services & State translation constraints & $\begin{array}{l}\text { The non-functional features of IoT } \\
\text { services are represented using state } \\
\text { translation constraints. }\end{array}$ \\
\hline has_Beh & $\begin{array}{l}\text { Environment } \\
\text { entities }\end{array}$ & Finite state machine & $\begin{array}{l}\text { The behavior of environment entities } \\
\text { are represented using FSM. }\end{array}$ \\
\hline has_Attr & $\begin{array}{l}\text { Environment } \\
\text { entities }\end{array}$ & State & $\begin{array}{l}\text { Each attribute of an environment entity } \\
\text { is mapped to a status in FSM. }\end{array}$ \\
\hline
\end{tabular}

行为.

3) 闭环系统原则. 物联网服务系统与物理环境的交互形成了一个由环境自主变化、环境变化触发 服务执行、服务执行改变环境状态的闭环系统. 整个环路具体表达如下:

(a) 运行时环境的生成. 相关的多个环境实体共同构成物联网系统的一个运行时环境. 该运行时 环境可通过自动机乘积的形式构成一个大自动机, 其中的每个状态是由环境实体的子状态构建而成.

(b) 环境实体的自主变化. 部分环境实体具有自主行为, 该行为无法被外界准确预知. 同时, 环境 实体之间有相互关联和影响, 单个环境实体的行为可能引起其它实体的状态变化. 环境实体的自主行 为、环境实体之间的交互共同导致了运行时环境的状态改变.

(c) 业务流程的触发. 运行时环境的状态被物联网服务系统中的感知设备实时监测, 环境的状态 变化将可能对应一些事件的发生, 如温度、烟雾浓度高于安全阈值对应着火灾的发生. 物联网服务系 统中的复杂事件侦测模块根据获取的实时环境信息, 判断是否有特殊事件发生, 以触发相应的物联网 业务流程的执行.

(d) 物联网服务的选择. 业务流程在执行过程中, 通过服务选择, 流程中的每个任务都将动态地与 某个物联网服务中的相应操作进行绑定, 即将抽象的流程转化为实际的服务操作. 


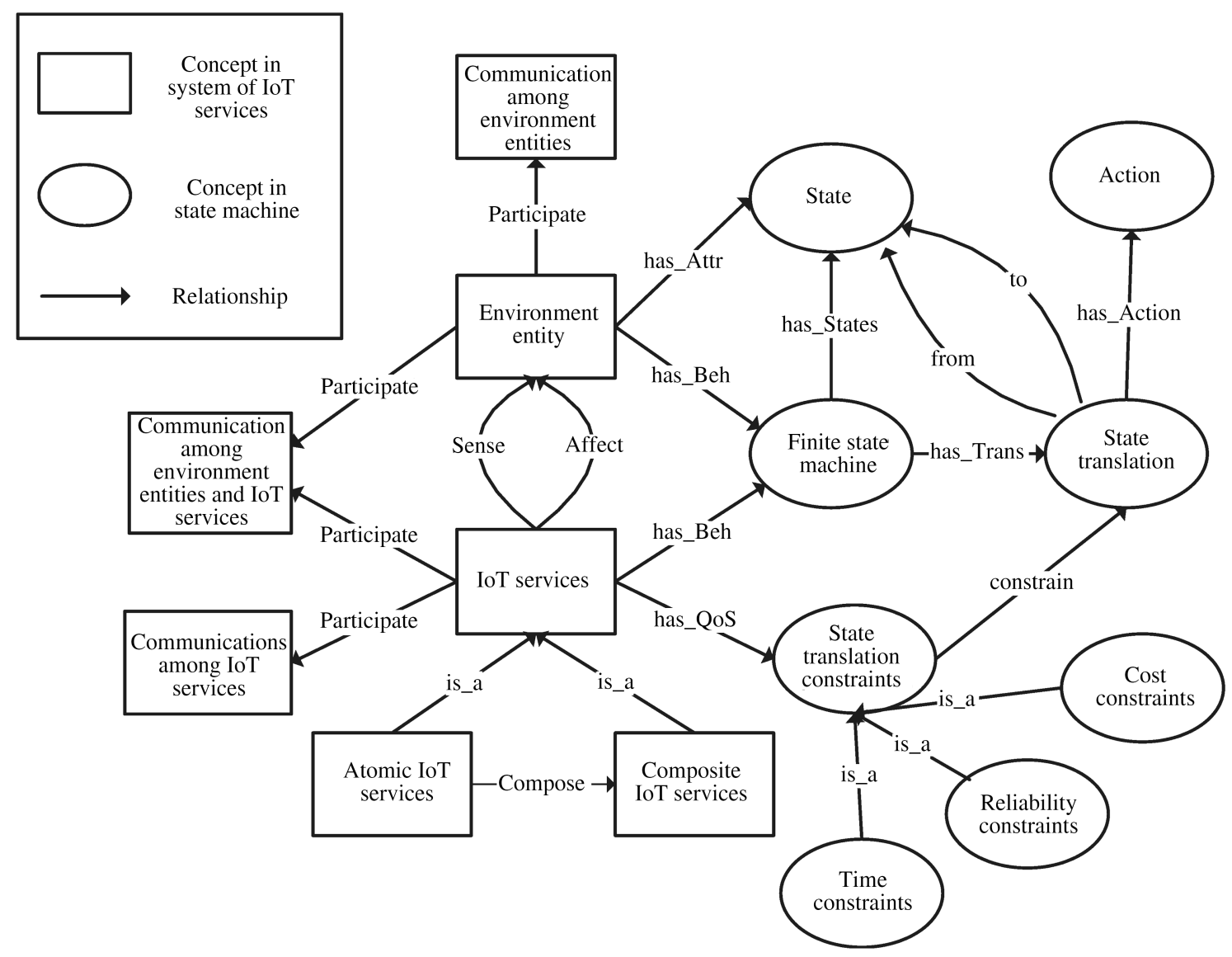

图 3 物联网服务系统本体的概念关联图

Figure 3 Concept relationship diagram of ontology for system of IoT services

(e) 物联网服务对环境的作用. 被选择的服务按照规定流程, 直接与环境实体进行交互, 控制环境 实体状态的变化, 运行时环境在物联网服务的作用下迁移到新的状态.

4) 需求驱动原则. 物联网服务在用户需求的驱动下, 行为随需而变, 作用于物理环境, 使其产生期 望的变化. 物联网服务系统的需求分为功能需求和非功能需求. 功能需求是指系统相关者对物联网服 务系统的期望, 期望它完成的任务或具备的功能, 具体体现在其对物理环境所产生的影响; 非功能需 求是指物理环境对物联网服务的行为约束, 如时间约束、性能约束、能耗约束等.

\section{4 物联网服务建模过程}

在上文给出的物联网服务本体的基础上，该小节提出一个基于环境的物联网服务建模过程，如 图 4 所示. 该建模过程主要包括以下几个步骤:

\section{- 识别给定应用场景中的服务和环境实体}

对给定的物联网具体应用场景, 根据物联网服务本体, 识别出场景中需要描述的数据对象. 该建模 过程主要强调对以下三类数据对象的描述: 物联网服务, 物理环境实体以及期望的性质. 


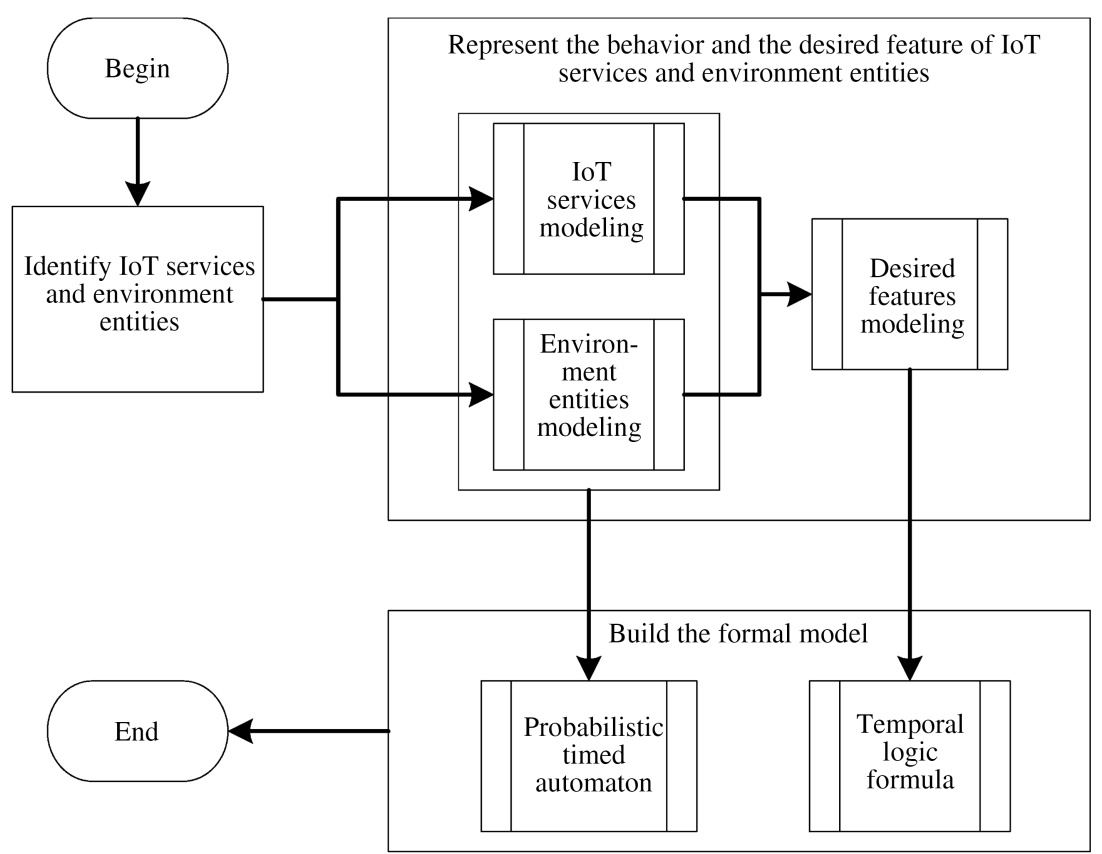

图 4 物联网服务建模过程

Figure 4 Process of IoT services modeling

\section{- 利用特定建模语言描述服务、环境实体的行为, 非功能属性以及期望的性质}

利用我们之前定义的物联网服务建模语言 IoTSML ${ }^{[15]}$ 分别对识别出的物联网服务的功能行为和 服务质量属性, 环境实体的功能行为以及期望的性质进行描述.

\section{- 建立形式化模型}

将上述物联网服务, 物理环境以及期望性质的描述转换为相应的形式化模型.

下面主要对上述建模过程中的后两个步骤进行详细描述.

\section{1 物联网原子服务的描述}

物联网服务描述主要从服务功能行为和服务质量属性两方面进行描述. 服务功能行为描述主要包 括服务的静态数据和动态行为两个部分. 其中, 静态数据部分需要描述服务感知或作用的环境实体集 合, 服务相关属性集合以及服务的接口; 动态行为部分描述服务的状态迁移, 即服务状态的变化. 在物 联网服务功能行为建模的基础上, 服务质量描述需要刻画服务动作的属性和约束, 具体包括三类质量 属性描述: 时间属性, 可靠性和资源消耗. 时间属性描述在物联网服务行为中所有与时间相关的约束; 可靠性描述物联网服务中每一个动作成功执行的概率; 资源消耗主要描述服务在各个状态停留所占用 的资源量, 以及状态迁移过程中所消耗的资源量.

在 IoTSML 语言中, 物联网原子服务的简要语法如表 2 所示, 完整语法详见 [15].

原子服务 $\langle$ AtomService $\rangle$ 由 4 个部分组成:

1) $\langle A S I D\rangle$ 表示每个物联网服务的唯一标识, 该标识由英文字符和数字组合而成.

2) $\langle S D a t a\rangle$ 表示物联网服务的静态数据部分, 其中, $\langle$ ESet $\rangle$ 表示与服务交互的环境实体类型的集 合; $\langle S A t t r\rangle$ 表示服务相关属性名的集合, 这些属性中既有静态属性, 又包含动态属性如位置、能耗信 息等; $\langle S O p\rangle$ 为服务可做的所有动作, 即服务的接口描述. 
表 2 IoTSML 关于原子服务描述的简要语法

Table 2 Statement of an atomic IoT service in IoTSML

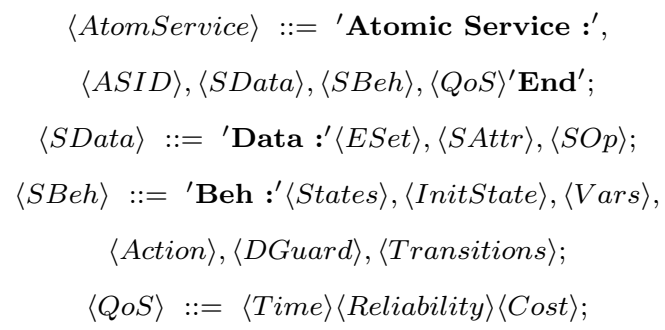

表 3 IoTSML 关于环境描述的简要语法

Table 3 Statement of the IoT environment in IoTSML

\begin{tabular}{c}
$\langle$ Environment $\rangle::={ }^{\prime}$ Environment $:^{\prime},\langle\text { EntityTypes }\rangle^{*}\langle\text { Entities }\rangle^{*} ;$ \\
$\langle$ EntityTypes $\rangle::=$ 'Entity Type $:^{\prime},\langle$ ETID $\rangle\langle$ EAttr $\rangle\langle$ EOp $\rangle ;$ \\
$\langle$ Entities $\rangle::={ }^{\prime}$ Entity $:^{\prime},\langle$ Entity $\rangle ;$ \\
$\langle$ Entity $\rangle::=\langle$ EID $\rangle\langle$ EType $\rangle\langle$ EBeh $\rangle ;$ \\
\hline
\end{tabular}

3) $\langle S B e h\rangle$ 表示物联网服务的动态行为部分. 关于服务动态行为的描述实际上可理解为状态迁移 系统 (State transition systems) 或有限自动机 (Finite automata), 其中,

- $\langle$ States $\rangle$ 表示一组状态的集合, 其中一个状态被指定为初始状态 $\langle$ InitState $\rangle$;

- $\langle$ V ar $s\rangle$ 表示数据变量的集合, 当中包含服务的属性集合 $\langle S A t t r\rangle$;

- $\langle$ Action $\rangle$ 为服务动作的集合;

- $\langle$ DGuard $\rangle$ 称为数据变量卫式 (guard), 由数据变量集合、逻辑运算符 $\wedge$ 、基本运算符 $(+,-, \times, \div)$ 和二元比较运算符 $(<,>, \leqslant, \geqslant)$ 按一定语法组合而成;

- $\langle$ Transitions $\rangle$ 定义迁移关系, 每一个迁移关系表示服务从某一个源状态开始执行, 若满足一定 的卫式, 则允许进行特定动作, 最终进入目的状态.

4) $\langle Q o S\rangle$ 表示服务质量属性相关部分. $\langle$ Time $\rangle,\langle$ Reliability $\rangle,\langle$ Cost $\rangle$ 分别表示物联网服务的时间 属性, 可靠性以及资源消耗属性.

\section{2 物理环境实体的描述}

在物联网系统中, 物联网服务的物理环境由与其进行交互的不同类型的环境实体共同构成. 环境 实体是指物理环境中所有可标识的、具有一定物理和/或虚拟属性的、可被服务感知或控制的实体, 它 们是物联网服务在物理世界中的交互对象和作用目标. 对每个环境实体的描述, 既含有用于强调实体 自身属性和实体间组织结构的数据描述部分, 又具有用于强调实体动态性质的行为描述部分. 其中, 数据描述部分包括属性及其取值, 所允许的操作以及所属的环境实体类别.

IoTSML 中关于环境实体及其类别的简要语法如表 3 所示, 完整语法详见文献 [15].

对于物理环境〈Environment $\rangle$ 的描述, IoTSML 要求先定义出环境实体类别, 再刻画具体的环境 实体. 其中,

1) $\langle$ EntityTypes $\rangle$ 表示环境实体类别. 其中, $\langle E T I D\rangle$ 是环境实体类别的唯一标识符; $\langle E A t t r\rangle$ 表 示这类环境实体所具有的属性名称, 如位置、大小、形状信息等; $\langle E O p\rangle$ 表示这类环境实体可能发生 的动作名称, 如移动、开启/关闭等. 
表 4 IoTSML 关于期望性质描述的简要语法

Table 4 Statement of the expected properties of IoT system in IoTSML

$\langle$ Properties $\rangle::={ }^{\prime}$ Properties :',' Functional' $\langle$ FuncProp $\rangle$

'Non-Functional' $\langle$ NonFuncProp $\rangle$;

$\langle$ FuncProp $\rangle::=\langle$ StateProp $\rangle \mid\langle$ StateProp $\rangle\langle$ FuncProp $\rangle$;

$\langle$ StateProp $\rangle::=\langle$ AtomProp $\rangle \mid{ }^{\prime} \operatorname{not}^{\prime}\langle$ StateProp $\rangle \mid$

$\langle\text { StateProp }\rangle^{\prime}(\text { and } \mid \text { or } \mid \text { imply })^{\prime}\langle$ StateProp $\rangle \mid$

'A' $\mathbf{A}^{\prime}\langle$ PathProp $\rangle \mid$ ' $\mathbf{E}^{\prime}\langle$ PathProp $\rangle$;

$\langle$ NonFuncProp $\rangle::=$ 'Time :' $\langle$ TimeProp $\rangle$

'Reliability :'〈ReliabProp $\rangle,$ 'Cost :'〈CostProp $\rangle$;

2) $\langle$ Entities $\rangle$ 表示环境实体, 由三部分构成: $\langle E I D\rangle$ 为环境实体的唯一标识符; $\langle E T y p e\rangle$ 记录一个 环境实体类别, 即环境实体所属的类别; $\langle E B e h\rangle$ 表示环境实体的动态行为, 其描述与物联网原子服务 一样, 也被表示为状态迁移系统, 同时还关注环境实体与时间相关的行为和约束.

\section{3 用户期望的描述}

期望性质也称为用户需求, 描述的是系统用户期望的物理环境和物联网服务在交互过程中所满足 的性质, 以及对服务行为的约束. 期望的性质分为功能性质和非功能性质两类. 其中, 功能性质描述用 户期望的物理环境实体状态变迁, 以及影响这些变迁的物联网服务的行为; 非功能性质描述依附于功 能性质之上, 用于描述用户期望的服务质量需求, 说明对环境和服务交互行为的约束, 比如时间约束、 资源约束等.

在 IoTSML 语言中, 对物联网服务系统所期望的性质以类似经典时序逻辑 CTL(computation tree $\operatorname{logic})^{[16]}$ 的方式表达, 其简要语法见表 4 , 完整语法详见文献 [15].

期望的性质 $\langle$ Properties $\rangle$ 主要包含功能性质和非功能性质两方面:

1) $\langle$ FuncProp $\rangle$ 表示功能性质, 在上文中物联网服务和环境实体的动态行为均表示为有限状态机, 环境和服务在状态变迁过程中所产生的状态序列称为路径 (path). 用户期望的功能性质可通过状态性 质和路径性质结合来表达. 其中,

(a) $\langle$ StateProp $\rangle$ 表示状态性质, 用于描述环境和服务的当前状态. 其中, 原子性质 $\langle$ AtomProp $\rangle$ 表 示服务或环境实体处于特定状态. 终结符 not, and, or, imply 表示状态性质之间的基本逻辑连接. $\mathbf{A}\langle$ PathProp $\rangle$ 表示从当前状态出发的所有可能路径, 均满足某个特定的路径性质; $\mathbf{E}\langle$ PathProp $\rangle$ 则表 示从当前状态出发的路径中至少有一条能满足特定路径性质.

(b) $\langle$ PathProp $\rangle$ 表示路径性质, 针对的是某条路径中包含的所有状态.

2) 〈NonFuncProp $\rangle$ 表示非功能性质. 其中, 〈TimeProp 表示时间相关性质, 即约束服务或环境 的动作必须在特定时间区间内发生; 〈ReliabProp 〉为可靠性约束, 表达功能性质被满足的概率大小需 受到一定的阈值约束; $\langle$ CostProp $\rangle$ 为资源消耗约束, 表示功能性质被满足时, 服务所消耗的资源数量 需保证在一定范围内.

\section{4 形式化的模型规约}

引入几个重要的符号集合如下: 
Chan: 所有信道名的集合; Act: 所有动作的集合, 包含输入、输出和内部三类动作, 即 $A c t=$ $\{a ? \mid a \in C h a n\} \bigcup\{a ! \mid a \in C h a n\} \bigcup\{\tau\} ;$ Clock: 所有时钟变量的集合; Var: 所有数据变量的集合.

在任意时钟变量集合 $C \subseteq C l o c k$ 和数据变量集合 $V \subseteq V a r$ 的取值上可以建立一些约束条件, 统 称为卫式, 分别记为 $\Phi(C)$ 和 $\Phi(V)$, 它们由变量集合、逻辑运算符 $(\wedge, \vee, \neg)$ 、基本运算符 $(+,-, \times, \div)$ 和二元比较运算符 $(<,>, \leqslant, \geqslant)$ 按一定语法构成.

除了对变量取值进行约束外、还可以对变量进行赋值操作. 假设 $C \subseteq C l o c k, V \subseteq V a r$, 则其上的 赋值操作分别记为 $R(C)$ 和 $R(V)$. 其中, 时钟变量只有置零赋值操作, 即 $c \in C, c:=0$, 而数据变量的 赋值操作形式为 $v \in V, v:=e, e$ 可为常量或各种运算表达式.

对于物联网服务而言, 服务质量部分的描述和分析同行为部分同样重要, 在 IoTSML 中也包含了 时间属性、可靠性、资源消耗等服务质量属性的描述. 更进一步地, 这些服务质量属性建立于行为描 述之上, 并且约束着服务的行为. 因此, 将 IoTSML 中关于物联网服务的行为和服务质量描述解释到 同一模型中, 是一种最为自然而合理的语义表示方法. 本文采用的建模工具为概率时间自动机.

概率时间自动机 $[17,18]$ 在时间自动机的基础上增加了离散概率分布, 可同时有效地表达系统行为 所具有的概率性、不确定性和连续时间.

给定概率时间自动机 $P T A=\left(S, s_{0}, A, C, V, I n v, E\right)$, 格局 (configuration) 用于描述概率时间自动 机的运行时状态, 每个格局表示为一个三元组 $\langle s, \rho, v\rangle$, 其中 $s \in S, \rho$ 表示对 $C$ 中所有时钟变量的一 个赋值, 且 $\rho$ 满足 $\operatorname{Inv}(s), v$ 表示对 $V$ 中所有数据变量的一个赋值. 直观上, 概率时间自动机的行为 可理解如下:

自动机的初始格局为 $\left\langle s_{0}, 0, v\right\rangle$, 即初始时, 处于状态 $s_{0}$, 且所有时钟变量被置为 0. 对于任意格局 $\langle s, \rho, v\rangle$, 其可能的行为有:

1) 动作迁移. 不确定性地选择出一条概率有向边 $(s, g, a, p) \in E$, 其中 $p$ 表示一个变量赋值操作 与状态组合的概率分布函数, 要求 $\rho$ 与 $v$ 满足卫式 $g$. 若 $r \in R(C) \wedge R(V), s^{\prime} \in S$, 且 $p\left(r, s^{\prime}\right)>0$, 则 自动机进行动作 $a$, 以概率 $p\left(r, s^{\prime}\right)$ 迁移到状态 $s^{\prime}$, 并根据赋值操作 $r$ 对变量的取值进行修改.

2) 时间流逝 (time pass). 状态 $s$ 与数据变量赋值 $v$ 不发生变化, 将 $V$ 中所有时钟变量的取值增 加数值 $t$, 且要求对于任意 $t^{\prime} \leqslant t$, 所有时钟变量的取值增加 $t^{\prime}$ 后, 依然满足 $\operatorname{Inv}(s)$.

概率时间自动机还可扩展代价结构 (cost structures), 用于刻画系统在运行时在特定状态停留或做 特定动作所需消耗的代价. 概率时间自动机 $P T A=\left(S, s_{0}, A, C, V, I n v, E\right)$, 为其扩展代价结构后, 表 示为 $\left(S, s_{0}, A, C, V\right.$, Inv $\left., E,\left(R_{\text {state }}, R_{\text {act }}\right)\right)$, 其中, $R_{\text {state }}: S \rightarrow \mathbb{R}_{\geqslant 0}$ 为状态代价函数, 表示处于某一状态 $s \in S$ 时, 每单位时间所需的代价; $R_{\mathrm{act}}: E \rightarrow \mathbb{R}_{\geqslant 0}$ 为动作代价函数, 表示做某一动作迁移所需的代价.

将物联网服务表示中的功能行为部分和附加的服务质量属性统一起来, 以概率时间自动机作为建 模工具, 就得到了服务的全局行为模型, 这也正是 IoTSML 语言关于原子服务行为描述的语义.

假设给定一个物联网服务的抽象表示, 其中行为部分为

$$
S B e h=\left(S, s_{0}, V, A, \text { Trans }\right) .
$$

服务质量部分为

$$
\text { NonFunc }=((\text { C, Invar, TGuard, CReset }), \text { Succ, Cost }) .
$$

则该服务的全局行为模型为一个带代价结构的概率时间自动机

$$
\text { GSBeh } \equiv\left(S, s_{0}, V, A, C, \text { Invar, GTrans, } \text { Cost }\right)
$$


其中, GTrans $\subseteq S \times(\Phi(V) \wedge \Phi(C)) \times A \times \operatorname{Dist}(S \times(\Phi(V) \wedge \Phi(C)))$ 是概率迁移关系的集合:

若有 $t=\left(s, g, a, s^{\prime}, r\right) \in$ Trans, 且 TGuard $(t)=g^{\prime}$, CReset $(t)=r^{\prime}$, 则 $\left(s, g \wedge g^{\prime}, a, p\right) \in G$ Trans, 其中, 概率分布 $p$ 定义为

$$
p\left(s^{\prime \prime}, r^{\prime \prime}\right)= \begin{cases}\operatorname{Succ}(t), & \text { 如果 } s^{\prime \prime}=s^{\prime}, r^{\prime \prime}=r \wedge r^{\prime}, \\ 1-\operatorname{Succ}(t), & \text { 如果 } s^{\prime \prime}=s, r^{\prime \prime}=r \wedge r^{\prime}, \\ 0, & \text { 否则. }\end{cases}
$$

根据上述定义可知, 服务的全局行为模型是在行为部分的基础上, 在时间相关属性方面, 以不变 量约束服务在特定状态的停留时间, 并为转移边增加相应的时钟卫式和时钟变量重置, 从而进一步表 达出每个动作发生的时间约束; 在可靠性方面, 根据动作迁移能否成功执行的概率, 随机地选择出后 继状态; 在资源消耗方面, 以代价结构来表达状态和动作迁移所花费的各项资源. 所有这些因素通过 形式化表达, 共同构成了一个带代价结构的概率时间自动机. 该自动机中的每一个格局, 代表着服务运 行时的一个状态. 根据概率时间自动机的操作语义, 格局之间的迁移序列就刻画出了物联网原子服务 在运行中的动作序列及状态变化的过程.

采用类似于上面的方法, IoTSML 中有关环境实体的描述同样可被解释为概率时间自动机, 实际 上这是一种简化的概率时间自动机即时间自动机 (timed automata). 而由多个环境实体所共同组成的 运行时环境也同样被建模为概率时间自动机网络.

在 IoTSML 中, 期望性质的语义通过时序逻辑公式来表达, 其中功能性质可完全使用 CTL 公式 来解释，而附着于功能性质之上的时间相关性、可靠性、资源消耗等则分别通过 CTL 的时间扩展 TCTL(Time CTL) $)^{[19]}$ 和概率扩展 PCTL(Probabilistic CTL) ${ }^{[20]}$ 来解释.

在上述形式化模型和逻辑公式解释的基础上, 即可以借助模型检测工具 UPPAAL ${ }^{[21]}$ 和 PRISM ${ }^{[22]}$ 进行需求的可满足性验证 ${ }^{[15]}$.

\section{5 建模过程小结}

该建模过程将传统软件建模方法中由软件系统、用户需求组成的二元问题域, 扩展为由物联网服 务、物理环境、期望的性质组成的三元问题域. 其中, 物联网服务和物理环境的交互正体现出物联网系 统的目标一信息世界与物理世界的无缝融合.一方面, 物联网服务的行为可以在其运行环境中被观察 与评估, 服务行为的作用结果将映射到其所处的环境之上, 另一方面, 物理环境的变化是由环境实体 的自主行为以及物联网服务对环境实体的控制所共同引起的. 该建模过程的主要优点如下:

1) 整体建模过程独立于任何特定的软件和硬件平台, 不关注具体的实现, 具有高度抽象性, 同时 将重点放在物联网服务之间通信和并发行为的描述.

2) 强调服务质量属性的建模. 对物联网服务的行为描述, 不仅关注其功能行为, 而且强调依附于 功能之上的多种服务质量属性的表示.

3) 关注物理环境. 物联网服务的行为能力通过与物理环境的交互体现出来, 需要在特定的环境中 进行合适的行为, 即必须同环境的变化适配. 该建模过程并不是对物联网服务进行孤立的建模, 而是 包含了对环境的刻画, 强调服务与环境的交互.

4) 显示描述期望的性质. 系统用户对物联网服务系统期望的性质是指受控的物理环境和服务的 状态变迁, 以及附加在这些变迁行为上的约束. 在该建模过程中, 系统期望的性质以一种形式化的、无 二义性的方式来陈述. 这样使得用户需求表达简洁而严格, 更易被建模者识别、理解和分析. 
5) 具有严格的形式化模型支持. 建模过程中关于物联网原子服务功能行为的描述被解释为有限 自动机, 而被附加上服务质量属性之后的全局行为则建模为带代价结构的概率时间自动机. 在此之上, 物联网组合服务被严格解释为概率时间自动机网络. 这些精确、严格的形式化模型为下一步的物联网 服务性质分析和验证打下了坚实的基础.

\section{5 案例研究}

本节以智能会议室应用场景为例, 对上文给出的物联网服务建模过程进行解释.

首先, 识别出物联网服务与环境实体, 实例化本体.

- 原子服务: 在智能会议室场景中与室内温度相关的原子服务有:

—气温感知服务, 用于采集室内实时温度, 表示为atomService(tempSensor);

一 空调控制服务, 用于向空调发出控制指令, 表示为atomService(acControl);

一温度调节服务, 用于对实时温度信息进行分析, 并作出相应的空调控制策略, 表示为atomService(tempProcess).

- 组合服务:一个调节室内温度的组合服务, 表示为compositeService(comp), 它是由上述 3 个 原子服务组合而成, 具体表示如下:

compose(tempSensor, comp), compose(acControl, comp), compose(tempProcess, comp)

- 环境实体: 在智能会议室场景中与室内温度相关的环境实体包括:

一空气实体, 表示为环境实体概念的实例 environEntity(air);

一空调实体, 表示为环境实体概念的实例 environEntity(ac).

- 环境实体的属性:

一空气实体具有温度属性, 表示为 has_Attr(air, temp);

— 空调实体具有运行状态属性, 表示为 has_Attr(ac, runningState).

- 服务对环境实体的感知和作用:

— 气温感知服务获取空气实体的温度信息, 表示为 sense(tempSensor, air);

一 空调控制服务直接控制空调实体的运行状态, 表示为 affect(acControl, ac).

然后, 利用 IoTSML 语言描述物联网服务, 环境实体以及期望的性质. 分别以空气温度感知服务 和空气实体描述来分别解释物联网服务和环境实体的描述. 一个空气温度感知服务的 IoTSML 描述 见表 5, 其中 Air 表示空气实体类, 该服务通过输入动作 $T e m p$ ? 采集气温信息, 并且可通过输出动作 GetTemp! 将该温度信息发送给其它服务, 在感知气温时服务可能发生故障, 进入到 FAULT 状态. 故 障发生的概率为 $5 \%$ (如 Reliability 中的描述). 服务每隔 10 个单位时间采集一次气温 (如 Invariant 和 Time guard 中的描述), 且每次数据采集消耗 20 个单位能量, 每次发送信息消耗 40 个单位能量 (如 Cost 中的描述).

空气环境实体类别 Air 和属于该类别的一个实体 air 的描述如表 6 所示, 该实体用变量 temp 和 humidity 分别存储温度和湿度, 通过信道 showTemp 和 showHumi 将温度、湿度值告知外界, 并每 隔 20 个单位时间内使用 $\tau$ 动作改变一次状态值.

对于一个智能会议室系统, 用户部分期望的性质包括:

1) 功能性质: 
表 5 气温感知服务的 IoTSML 描述

Table 5 IoTSML statement of the temperature sensing service

Atomic service: TempSensor

Data :

Environment : Air;

Attr : temp $\rightarrow$ Real;

Op : Temp, GetTemp;

Beh :

States : $R U N, F A U L T$;

Initial state : $R U N$;

Variables : temp, in, out;

Actions : Temp?,GetTemp!;

Transitions :

$$
\begin{aligned}
& t 1: R U N,\{\}, \text { Temp? } \rightarrow \text { RUN, }\{\text { temp }:=i n\} ; \\
& t 2: R U N,\{\}, \text { Temp? } \rightarrow \text { FAULT, }\{\} ; \\
& t 3: R U N,\{\}, \text { GetTemp } ! \rightarrow R U N,\{\text { out }:=\text { temp }\} ; \\
& t 4: F A U L T,\{\}, \tau \rightarrow R U N,\{\} ;
\end{aligned}
$$

Clocks : c

Invariant $: R U N \rightarrow\{c \leqslant 10\}$

Time guard : $t_{1} \rightarrow\{c==10\}, t_{2} \rightarrow\{c==10\} ;$

Clock reset : $t 1 \rightarrow\{c:=0\}, t 4 \rightarrow\{c:=0\}$;

Reliability :

$t 1 \rightarrow 0.95, t 2 \rightarrow 0.05, t 3 \rightarrow 1, t 4 \rightarrow 1 ;$

Cost :

$t 1 \rightarrow 20, t 3 \rightarrow 40$

End

(a) 安全性:

$p 1$ : 室内温度被恒定控制在 15 至 25 摄氏度之间;

$p 2$ : 室内的温度感知服务 1 和温度感知服务 2 不会同时失效.

(b) 活性:

$p 3$ : 若室内温度高于 25 摄氏度, 则空调将启动并进入制冷状态;

$p 4$ : 若室内投影仪开启, 则日光灯将自动关闭.

2) 非功能性质:

(a) 时间约束: 性质 $p 3$ 中空调的反应时间在 60 秒以内;

(b) 可靠性约束: 性质 $p 2$ 被满足的概率高于 $90 \%$;

(c) 资源消耗约束: 满足性质 $p 1$ 的平均能量消耗在 100 个能量单位内.

上述期望性质的 IoTSML 描述如表 7 所示.

最后, 建立形式化模型. 将表 5 中的气温感知服务描述和表 6 中的空气实体描述转化为概率时间. 
表 6 空气实体的 IoTSML 描述

Table 6 IoTSML statement of the entity of room air

\section{Environment}

Environment Type: Air;

Attr : loc $\rightarrow$ Loction, temp $\rightarrow$ Real, humidity $\rightarrow$ Real;

Op : showTemp, showHumi;

Environment Entity: air;

Type : Air;

Beh :

States : $S$

Initial State : $S$;

Variables : $\{x$, temp, humidity $\}$;

Actions : showTemp!, showHumi!, $\tau$;

Transitions :

$$
\begin{aligned}
& t 1: S,\{\}, \text { showTemp } ! \rightarrow S,\{x:=\text { temp }\} \\
& t 2: S,\{\}, \text { showHumi } ! \rightarrow S,\{x:=\text { humidity }\} \\
& t 3: S,\{\}, \tau \rightarrow S,\{\text { temp }:=\text { newTemp, humi }:=\text { newHumi }\}
\end{aligned}
$$

Clocks : c

Invariant $: S \rightarrow\{c \leqslant 20\}$

Time Guard : $t 3 \rightarrow\{c \leqslant 20\}$;

Clock Reset : $t 3 \rightarrow\{c:=0\}$

End

表 7 智能会议室期望性质的 IoTSML 描述

Table 7 IoTSML statement of the expected properties in smart meeting room

\section{Properties}

\section{Functional:}

$p 1: \mathbf{A} \mathbf{G}$ (air.temp $\geqslant 15$ and air.temp $\leqslant 25) ;$

$p 2: \mathbf{A} \mathbf{G}($ not (TempSensor1.FAULT and TempSensor $2 . F A U L T)$ );

$p 3: \mathbf{A} \mathbf{G}$ (air.temp $>25$ imply A F (ac.COOL));

$p 4:$ A G (projector.ON imply A F (light.OFF));

Non-Functional:

Time: $p 3 \rightarrow(c<60)$

Reliability: $p 2>0.9$

Cost: $p 1<100$

End

自动机模型分别如图 5 和图 6 所示.

将表 7 中的智能会议室期望性质描述转化为时序逻辑公式如下所示: 


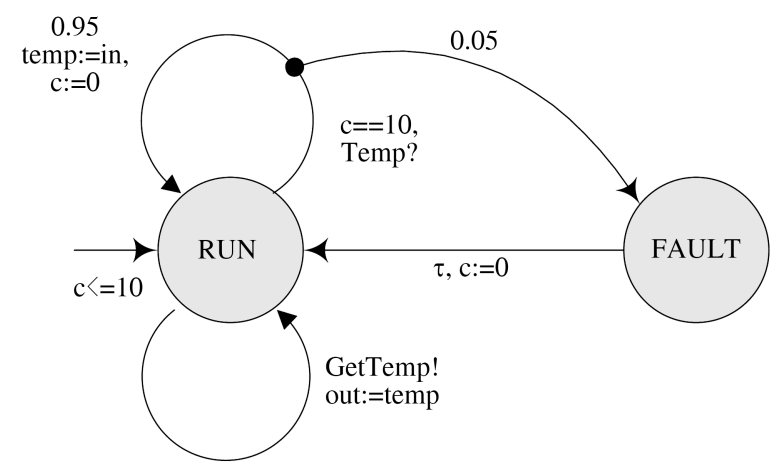

图 5 气温感知服务的概率时间自动机描述

Figure 5 Probabilistic timed automata of the temperature sensing service

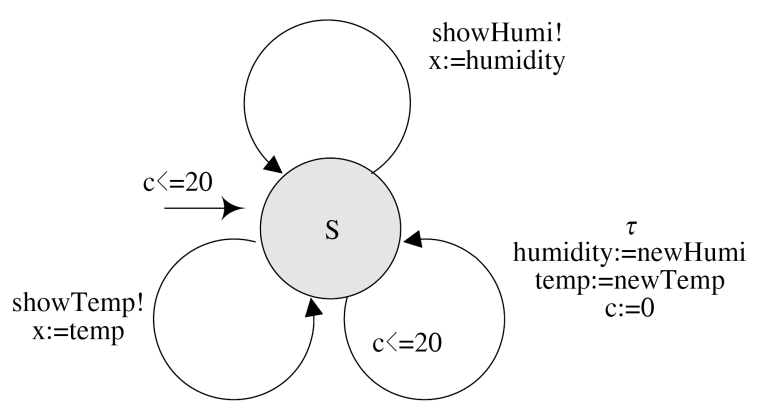

图 6 空气实体的概率时间自动机描述

Figure 6 Probabilistic timed automata of the entity of room air

$p 1: \forall \square($ air.temp $\geqslant 15 \wedge$ air.temp $\leqslant 25)$,

$p 2: P_{>0.9}[\forall \square(\neg($ tempSensor $1 . F A U L T \wedge$ tempSensor $2 . F A U L T))]$,

p3: $\forall \square($ air.temp $>20 \rightarrow \forall \diamond($ ac.COOL $\wedge$ air.c $<60))$,

$p 4: \forall \square($ projector.ON $\rightarrow \forall \diamond$ light.OFF $)$.

借助经典模型检测工具 UPPAAL 和 PRISM, 便可以验证物联网服务模型是否能满足期望的 性质 ${ }^{[15]}$.

\section{6 结论}

本文首先归纳出物联网服务的特点, 然后结合基于环境建模的需求工程思想, 提出基于环境的物 联网服务建模框架. 该框架强调物联网服务行为描述中的物联网环境. 在此基础上, 本文设计出一个 物联网服务本体, 以规范框架中的概念和关联关系, 并总结出一组建模原则. 更进一步地, 本文构建出 一种物联网服务建模过程, 该建模过程不仅包含对物联网服务的描述, 而且还强调对物理环境的刻画, 以及服务与环境的交互. 它在关注服务功能行为的同时, 对多种服务质量属性 (时间属性、可靠性、资 源消耗) 均有相应的建模. 此外, 该过程还具有基于概率时间自动机和时序逻辑的形式化模型解释, 从 而为各种服务性质分析和验证奠定了基础. 
由于物联网服务所处的环境具有动态变化性, 物联网服务之间的调用关系易发生变化. 因此, 下 一步工作将强调如何对物联网服务的动态调用关系进行建模, 并通过分析获取相对可靠、稳定的服务 提供; 其次, 各类物联网终端设备在提供丰富信息的同时, 也增加了信息暴露的风险. 所以如何对物联 网服务行为的安全性和私密性进行建模和分析也是我们下一步的研究工作.

\section{参考文献}

1 Liu Y H. Introduction to Internet of Things. Beijing: Science Press, 2011. 3-10 [刘云浩. 物联网导论. 北京: 科学出版社, 2011. 3-10]

2 Deugd S, Carroll R, Kelly K, Millett B, Ricker J. SODA: service oriented device architecture. IEEE PERVAS COMPUT, 2006, 5: 94-96

3 Buckl C, Sommer S, Scholz A, et al. Services to the field: an approach for resource constrained sensor actor networks. In: Proceedings of Workshop on Service Oriented Architectures in Converging Networked Environments. Bradford: IEEE Trans SERV COMPUT, 2009. 476-481

4 Guinard D, Trifa V, Karnouskos S, et al. Interacting with the SOA-based Internet of Things: discovery, query, selection, and on-demand provisioning of web services. IEEE Trans SERV COMPUT, 2010, 3: 223-235

5 Pautasso C, Zimmermann O, Leymann F. RESTful web services vs. big web services: making the right architecture decision. In: Proceedings of International Conference on World Wide Web. Beijing: ACM, 2008. 805-814

6 De S, Barnaghi P, Bauer M, et al. Service modeling for the Internet of Things. In: Proceedings of Federated Conference on Computer Science and Information Systems. Szczecin: COMPUT SCI INF SYST, 2011. 949-955

7 Li L, Jin Z, Li G. Modeling and Verifying Services of Internet of Things Based on Timed Automata, Chin J Comput, 2011, 24: 1356-1377 [李力行, 金芝, 李戈. 基于时间自动机的物联网服务建模和验证. 计算机学报, 2011, 34: 1356-1377]

8 Li L, Jin Z, Li G. Modeling and analyzing the reliability and cost of service composition in the IoT: a probabilistic approach. In: Proceedings of IEEE International Conference on Web Services. Honolulu: IEEE International Conference on Web Services, 2012. 584-591

9 Hou L S, Jin Z, Wu B D. Requirement Driven Web Service Modeling and Verifying. Sci China Ser-E Inf Sci, 2006, 36: 1189-1219 [侯丽珊, 金芝, 吴步丹. 需求驱动的 web 服务建模及其验证. 中国科学 E 辑, 2006, 36: 1189-1219]

10 Wang P, Jin Z, Liu L, et al. Building toward capability specifications of web services based on an environment ontology. IEEE Trans Knowl Data Eng, 2008, 20: 547-561

11 Liu Y, He Y, Li M, et al. Does wireless sensor network scale? a measurement study on GreenOrbs. In: Proceedings of IEEE Conference on Computer Communications. Shanghai: INFOCOM, 2011. 873-881

12 Helal S, Mann W, El-Zabadani H, et al. The Gator Tech smart house: a programmable pervasive space. Computer, 2005, 38:50-60

13 Zuehlke D. SmartFactory-towards a factory-of-things. Annu Rev Control, 2010, 34:129-138

14 Studer R, Benjamins V, Fensel D. Knowledge engineering, principles and methods. Data Knowl Eng, 1998, 25: 161-197

15 Li L X. Environment based Services Describing and Performance Analysing in Internet of Things, Dissertation for the Doctoral Degree, Beijing: University of Chinese Academy of Sciences, 2013 [李力行. 基于环境建模的物联网服务描述与 性能分析. 博士论文, 中国科学院研究生院, 2013]

16 Clarke E, Emerson E, Sistla A. Automatic verification of finite-state concurrent systems using temporal logic specifications. ACM Trans Program Lang Syst, 1986, 8: 244-263

17 Kwiatkowska M. Norman G, Segala R. Automatic verfication of real-time systems with discrete probability distributions. Theor Comput Sci, 2002, 282: 101-150

18 Kwiatkowska M. Norman G, Sproston J. Symbolic model checking for probabilistic timed automata. Inf Comput, 2007, 205: 1027-1077

19 Alur R, Courcoubetis C, Dill D. Model-checking in dense real-time. Inf Comput, 1993, 104: 2-34

20 Hansson H, Jonsson B. A logic for reasoning about time and reliability. Form Asp Comput, 1994, 6: 512-535

21 Larsen K, Petterson P, Wang Y. UPPAAL in a nutshell. International Journal on Software Tools for Technology Transfer, 1997, 1: 134-152 
22 Kwiatkowska M, Norman G, Parker D. PRISM 4.0: Verification of Probabilistic Real-time Systems. In: Proceedings of International Conference on Computer Aided Verification (CAV), Snowbird: Springer Berlin Heidelberg, 2011. 6806: $585-591$

\title{
Environment based modeling approach for services in the Inter- net of Things
}

\author{
LI Ge ${ }^{1,2}$, WEI Qiang ${ }^{3,4}$, LI LiXing ${ }^{3,4}$, JIN Zhi $^{1,2 *}$, XU Yan $^{3,4}$ \& ZHENG LiWei ${ }^{5}$ \\ 1 Software Institute, School of Electronic Engineering and Computer Science, Peking University, Beijing 100871, \\ China; \\ 2 Key Laboratory of High Confidence Software Technologies (Peking University), Ministry of Education, Beijing \\ 100871, China; \\ 3 Academy of Mathematics and System Science, Chinese Academy of Sciences, Beijing 100190, China; \\ 4 University of Chinese Academy of Sciences, Beijing 100049, China; \\ 5 Computer School, Beijing Information Science and Technology University, Beijing 100085, China \\ *E-mail: zhijin@sei.pku.edu.cn
}

\begin{abstract}
Services in Internet of Things (IoT services) are extensions of traditional Web services. Through sensor networks, IoT services can communicate directly with the entities in the physical environment, and even impact them directly. So, because of the influence of the physical environment (such as the time limitation, the resource limitation and the equipment fault probability), features of IoT services are more easily affected by the physical environment than the traditional Web service. In this case, the response time, the service consumption and the fault-tolerant ability become important factors in the performance of the whole IoT system. Therefore, modeling the IoT services detailedly and formally is very important for the analysis and prediction of the performance of the whole IoT system. In this paper, we propose an environment based IoT service modeling framework as well as an ontology of the IoT service and a triple-element model, and advance the corresponding modeling principle. Based on this framework, we proposed a detailed IoT model method, in which the IoT services and their corresponding environment can be described in probabilistic timed automata. The user's desired IoT services features can be described in the temporal logic formula. With our method, the validity of the whole system and the satisfiability of its non-functional constraints can be analyzed detailedly and formally.
\end{abstract}

Keywords Internet of Things (IoT), IoT services, environment based modeling, ontology of service, service modeling process 


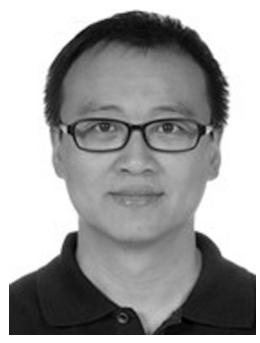

LI Ge was born in 1977. He received the $\mathrm{Ph}$. D. degree in Computer Science from Peking University, Beijing, in 2006. Currently, he is an Associate Professor at School of EE\&CS, Peking University. His research interest includes Software Engineering, Knowledge Engineering and Machine Learning. Dr. $\mathrm{Ge} \mathrm{Li}$ is a member of IEEE and China Computer Federation.

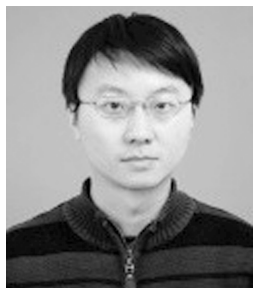

LI LiXing was born in 1983. He is Ph.D. candidate at Academy of Mathematics and Systems Science, the Chinese Academy of Sciences. His research interest includes service-oriented computing and formal methods.

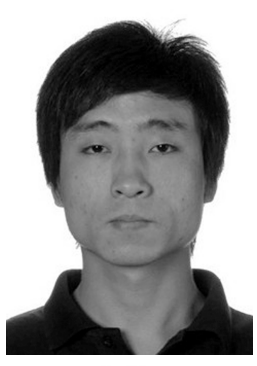

WEI Qiang was born in 1985. He is a Ph.D. candidate at Academy of Mathematics and Systems Science, the Chinese Academy of Sciences. His research interest includes service modeling and discovery in Internet of things.

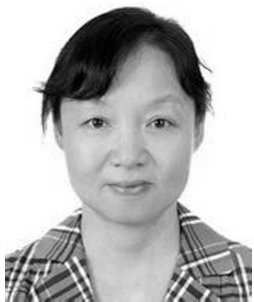

JIN Zhi was born in 1962. She received the Ph. D. degree in Computer Science from the National University of Defense Technology, Changsha, in 1992. Currently, she is a Professor at School of EE\&CS, Peking University. Her research interest includes Knowledge Engineering, Requirement Engineering and Artificial Intelligence. Dr. China Computer Federation. 